\title{
Dietary and lifestyle habits amongst adolescents in Bahrain
}

\author{
Abdulrahman O. Musaiger'*, Zahra Bader², Khaldoon \\ Al-Roomi ${ }^{3}$ and Reshma D'Souza ${ }^{4}$
}

'Nutrition and Health Studies Unit, Deanship of Scientific Research, University of Bahrain, Kingdom of Bahrain;

2 Ministry of Health, Kingdom of Bahrain; ${ }^{3}$ College of Medicine and Medical Science, Arabian Gulf University, Kingdom of Bahrain; ${ }^{4}$ Bahrain Centre for Studies and Research, Kingdom of Bahrain

Abstract

Background: Changes in dietary habits and lifestyle are considered the main factors associated with several diet-related diseases in the Arab Gulf countries. The aim of this study was, therefore, to describe the dietary and lifestyle habits amongst adolescents in Bahrain.

Design: A cross-sectional study was carried out amongst male and female secondary school students selected using the multi-stage stratified random sampling technique. A sample size of 735 subjects (339 males and 396 females), aged 15-18 years, was selected from government schools from all the governorates of Bahrain.

Results: Skipping breakfast was significantly greater in females $(62.8 \%)$ compared to males $(37.2 \%),(P<0.01)$. About $88 \%$ of adolescents snacked during school break, $70.7 \%$ procuring food from the school canteen. Fruit was not consumed by about $27.7 \%$ of respondents ( $33.5 \%$ males, $66.5 \%$ females) and the gender difference was statistically significant $(P<0.01)$. Fish and lentils were less preferred, while chicken was more popular. There was no significant difference between gender and frequency of eating fast food. About $8.4 \%$ of respondents reported not eating burgers, with $68.8 \%$ preferring regular size burgers. Furthermore, 24.4\% preferred large portions of potato chips ( $53.1 \%$ male, $46.9 \%$ female). About $29.8 \%$ watched TV for more than 5 hours a day $(51.2 \%$ females, $48.8 \%$ males). About $69 \%$ of males practiced sports everyday as against $30.8 \%$ of females $(P<0.01)$ and $81.6 \%$ of those who participated in sport activity outside school were males compared to $18.4 \%$ of females.

Conclusion: It seems that the adolescents in Bahrain are moving toward unhealthy dietary habits and lifestyles, which in turn will affect their health status in the future. Promoting healthy lifestyle and eating habits should be given a priority in school health programs.

Keywords: Bahrain; adolescents; food intake; fast foods; lifestyle; physical activity

Received: 23 March 201 I; Revised: 7 July 20I I; Accepted: 2 August 20 I ; Published: 9 September 20I I

$\mathrm{E}$ vidence shows that dietary quality declines from childhood to adolescence. The intake of fruit, vegetables, milk, and fruit juices decreases, whereas the intake of soft drinks increases during this time (1). Studies among American children indicate that the food choices of adolescents are not consistent with the dietary guidelines and food intake tends to be low in fruit, vegetables, and calcium-rich foods and high in fat (2). Hilsen et al. (3) reported that a large number of Norwegian adolescents consume unhealthy food items at school and very few eat fruit and vegetables. Studies on adolescent food intake in different European countries showed a similar consumption of fat, particularly saturated fat and low fiber intake. Animal sources of protein represented about two-thirds of total protein intake among adolescent girls with a low intake of calcium and iron (4).

With regard to the Arabian Gulf countries (including Bahrain), poor dietary habits such as skipping breakfast, a low intake of milk, fruits, and vegetables; and a high intake of carbonated beverages, sweets, and fast food were reported by several studies on school children (5-7). In addition, the significant epidemiological transformations that have occurred over the past two or three decades have resulted in the modification of the lifestyle to a more sedentary way of living, with considerable reduction in the extent of physical activity, an increase in the intake of fast foods, and the elevation of stress-related work and other activities (8). A study on Omani adolescent girls has indicated that overweight adolescent girls had a high 
intake of energy-rich foods (9). In general, studies in the Arabian Gulf countries have reported a high consumption of foods rich in fats and calories among most communities with the results of increasing the risk of obesity and its co-morbidities (10). In Bahrain, studies on dietary habits among adolescents are, at most, scanty. In 1992 Musaiger and Gregory (11) found that skipping breakfast and consumption of calorie-dense foods was quite common amongst Bahraini adolescents. However, the investigation did not report any information on the lifestyle habits of the adolescents.

In view of the above, it was considered of interest to find out the dietary habits and lifestyle patterns among Bahraini adolescent school children. This will provide a basis to develop suitable intervention programs that encourage healthy eating and lifestyle options for this age group.

\section{Methods and subjects}

This study is a part of a project on obesity and lifestyle among adolescents in Bahrain and the United Arab Emirates. The project is divided into three parts: anthropometric measurements and obesity, factors associated with obesity, and lifestyle of adolescents. The current study covered the third part, which related to lifestyle in this age group in Bahrain.

\section{Sampling}

This was a descriptive cross-sectional study consisting of male and female grade 1-3 secondary school students selected from government schools from all the governorates of Bahrain. The sample was calculated with the assumption of prevalence of $50 \%$ of unhealthy dietary habits, $90 \%$ confidence interval, and perception of 0.03 .

Students have been selected using a multi-stage stratified and clustered random sampling technique according to the gender, governorates, and grades. The final study sample size was 735, aged between 15 and 18 years (339 males and 396 females). The Kingdom of Bahrain is divided into five governorates: Capital, Al-Muharaq, North, Central, and South. Each governorate is served by a number of schools, which is proportional to the population density of a given governorate. The total number of secondary public schools was 30 (17 school for females and 13 for males) at the time of the study (2006). Male and female public secondary school children attend separate schools. A list of secondary schools in Bahrain was obtained from the Ministry of Education. Separate lists for males and females were used to obtain the sample; stratified by gender, governorate, and grade; and clustered on schools and class. The number of students chosen from each governorate was proportional to the student population density in that governorate.

The students were assembled in their classrooms. The objectives of the study as well as the questions in the questionnaire were explained to the students. Then the students were asked to fill in the questionnaire, which was self-administrated. All the students attending the chosen classes at the time of collecting the data participated in the study and therefore there were no non-responders.

\section{Ethical consideration}

Permission to conduct this research was obtained from the Education and Development Research Committee in the Ministry of Education, which in turn informed the principals of the schools, teachers, and students before conducting the study. Parents were informed about the study through the schools.

\section{Reliability and validity of the questionnaire}

The questionnaire for this study was modified from a previously validated questionnaire (12). The current questionnaire was reviewed by nutrition specialists at Bahrain Center for Studies and Research and Ministry of Health in order to check it in terms of the extent to which the items reflect the concept of identifying the dietary habits of adolescents. The content validity of the tool was then tested by five experts in the field of nutrition, public health, and epidemiology from the Ministry of Health, Arabian Gulf University, and Bahrain Center for Studies and Research to ensure that the items in the questionnaire represent the objectives and provide adequate coverage of the purpose of the study. They all generally agreed about the content and some changes were incorporated according to their suggestions.

The stability of the questionnaire has been evaluated by test-retest reliability. The time between test and pretest was 1 week. The questionnaire was administered to the students on two occasions during the pilot study. The comparison between the results of the first and the second questionnaire was obtained by computing Pearson correlation, the result showed the value of 0.87 for Pearson correlation, which indicates that the questionnaire used in the study was reliable.

\section{Pilot study}

Forty-eight students (22 male and 26 female students, one class from the male and the female secondary school) were employed in the pilot study by completing the questionnaire after obtaining their permission and explaining the objectives of the study. The pilot study assessed the items where the participants were reluctant to answer any questions. The questionnaire required 1520 min to be completed by the students. The final draft of the questionnaire had been modified based on the feedback received from the students. The two schools participating in the pilot study were excluded from the selected schools for the study. 


\section{The questionnaire}

The instrument selected to measure the identified variables (dietary habits, lifestyle, and physical activities) is a structured, self-administered questionnaire. The questionnaire was divided into two parts: the first part relates to socio-demographic data including age, sex, and educational status of parents. Parent's level of education was defined as low (illiterate, those who can read, write, and have primary school education), middle (intermediate and secondary school education), and high (university education).

The second part consists of different questions pertinent to students' dietary habits and lifestyle. Information related to physical activity such as frequency of playing sports inside and outside the school and participation in physical education classes was obtained through selfreported questions.

\section{Data analysis}

All the questionnaires were reviewed for completion immediately after administering them to the students in the schools. Data were entered directly from the precoded questionnaires. Computer printouts of the data were reviewed for any information that was out of range. The statistical analysis was undertaken using the Statistical Package Social Sciences (SPSS), version 12 relevant descriptive statistics, as well as inferential statistics were compiled. Chi-square tests were used to determine the presence of association between the variables.

\section{Results}

Socio-demographic characteristics of the adolescents that participated in the study are given in Table 1. There was no significant difference in age distribution between males and females. About $22 \%$ of the adolescents' fathers and $32 \%$ of their mothers had low educational level. A higher level of education for fathers $(31.2 \%)$ was observed as compared with that of the mothers $(25.2 \%)$.

The food consumption and snacking patterns of the Bahraini adolescents are shown in Table 2. About 56\% of the adolescents did not consume breakfast regularly, the proportion of females was nearly double $(62.8 \%)$ than the males $(37.2 \%)$, and the difference was statistically significant $(P<0.01)$. Similarly, the proportion of females who did not consume a regular lunch and supper (68.3 and $66.5 \%$, respectively) was twice that of the males (31.7 and $33.5 \%$, respectively) and the gender difference for both lunch and supper was statistically significant $(P<0.01)$.

On the whole about $88 \%$ of students consumed food during the school break. Most of them (70.7\%) ate from the school canteen, while $34.4 \%$ ate food brought from home but with no significant gender difference. Supper was less likely to be eaten regularly $(73.6 \%)$ than
Table 1. Socio-demographic characteristic of Bahraini adolescents

\begin{tabular}{|c|c|c|c|c|c|c|c|}
\hline \multirow[t]{3}{*}{ Socio-demographic } & \multicolumn{7}{|c|}{ Gender } \\
\hline & \multicolumn{2}{|c|}{ Total } & \multicolumn{2}{|c|}{ Male } & \multicolumn{2}{|c|}{ Female } & \multirow[b]{2}{*}{$P$-value* } \\
\hline & $N$ & $\%$ & $N$ & $\%$ & $N$ & $\%$ & \\
\hline Number (\%) & 735 & 100 & 339 & 46.1 & 396 & 53.9 & \\
\hline \multicolumn{8}{|l|}{ Age (years) } \\
\hline 15 & 220 & 29.9 & 90 & 26.5 & 130 & 32.8 & 0.054 \\
\hline 16 & 239 & 32.5 & 119 & 35.1 & 120 & 30.3 & \\
\hline 17 & 215 & 29.3 & 108 & 31.9 & 107 & 27.0 & \\
\hline 18 & 61 & 8.3 & 22 & 6.5 & 39 & 9.8 & \\
\hline \multicolumn{8}{|l|}{ Father's education } \\
\hline Low & 160 & 21.8 & 61 & 18.0 & 99 & 25.0 & 0.048 \\
\hline Middle & 346 & 47.1 & 162 & 47.8 & 184 & 46.5 & \\
\hline High & 229 & 31.2 & 116 & 34.2 & 113 & 28.5 & \\
\hline \multicolumn{8}{|l|}{ Mother's education } \\
\hline Low & 233 & 31.7 & 93 & 27.4 & 140 & 35.4 & 0.021 \\
\hline Middle & 317 & 43.7 & 147 & 43.4 & 170 & 42.9 & \\
\hline High & 185 & 25.2 & 99 & 29.2 & 86 & 21.7 & \\
\hline
\end{tabular}

*Chi-square test, the $p$-value indicates the comparison between males and females.

lunch $(83.3 \%)$. Males were more likely to eat lunch and supper on a regular basis than females. The difference was statistically significant $(P<0.001)$.

The type and frequency of food consumed by Bahraini adolescents are presented in Table 3 . About 25\% of the respondents reported eating fruit daily, while $27.7 \%$ consumed fruit rarely. Of those who rarely ate fruit, $33.5 \%$ were males and $66.5 \%$ were females. In general, males were more prone to consume fruit than females $(P<0.001)$. In addition, the proportion of respondents who rarely consumed vegetables was higher $(38.1 \%)$ than those who consumed vegetables everyday $(26 \%)$. Reverse trends were observed concerning the consumption of milk and other dairy products, where 37\% consumed these products daily with the proportion of females $(52.7 \%)$ more than males $(47.3 \% ; P<0.02)$. The gender difference was also significant with meat consumption $(P<0.001)$, as males were more likely to consume meat than females.

Only $6.9 \%$ of the participants consumed fish on a daily basis, while a relatively high proportion $(38.9 \%) \mathrm{did}$ so rarely. Fish consumption was significantly more favored by males than females $(P<0.003)$. Chicken was more popular among the study group, with $18.2 \%$ of the respondents consuming chicken every day, while lentils were less preferred (4.1\%). A similar preference was observed for nuts (7.6\% ate nuts daily) and canned fruit juice (23.9\% consumed daily). 
Table 2. Food consumption and snacking patterns of Bahraini adolescents

\begin{tabular}{|c|c|c|c|c|c|c|c|}
\hline \multirow[b]{2}{*}{$\begin{array}{l}\text { Food and snacking } \\
\text { pattern }\end{array}$} & \multicolumn{2}{|c|}{ Total } & \multicolumn{2}{|c|}{ Male } & \multicolumn{2}{|c|}{ Female } & \multirow[b]{2}{*}{$P$-value } \\
\hline & $N$ & $\%$ & $n$ & $\%$ & $n$ & $\%$ & \\
\hline \multicolumn{8}{|c|}{ Eating breakfast regularly } \\
\hline No & $4 I I$ & 55.9 & 153 & 37.2 & 258 & 62.8 & 0.000 \\
\hline Yes & 324 & 44.1 & 186 & 57.4 & 138 & 42.6 & \\
\hline \multicolumn{8}{|c|}{ Place of eating breakfast } \\
\hline At home & 448 & 61.0 & 203 & 45.3 & 245 & 54.7 & 0.001 \\
\hline At school & 287 & 39.0 & 136 & 47.4 & $|5|$ & 52.6 & \\
\hline \multicolumn{8}{|c|}{ Bring food from home } \\
\hline No & 482 & 65.6 & 284 & 58.9 & 198 & 41.1 & 0.000 \\
\hline Yes & 253 & 34.4 & 55 & 21.7 & 198 & 78.3 & \\
\hline \multicolumn{8}{|l|}{ Eating during school } \\
\hline \multicolumn{8}{|l|}{ break time } \\
\hline No & 88 & 12.0 & 49 & 55.7 & 39 & 44.3 & 0.057 \\
\hline Yes & 646 & 88.0 & 290 & 44.9 & 356 & 55.1 & \\
\hline \multicolumn{8}{|c|}{ Eat from school canteen } \\
\hline No & 215 & 29.3 & 104 & 48.4 & III & 51.6 & 0.431 \\
\hline Yes & 520 & 70.7 & 235 & 45.2 & 285 & 54.8 & \\
\hline \multicolumn{8}{|c|}{ Eating food while going } \\
\hline \multicolumn{8}{|l|}{ home } \\
\hline No & 580 & 78.9 & 257 & 44.3 & 323 & 55.7 & 0.057 \\
\hline Yes & 155 & 21.1 & 82 & 52.9 & 73 & 47.1 & \\
\hline \multicolumn{8}{|l|}{ Eating lunch regularly } \\
\hline No & 123 & 16.7 & 39 & 31.7 & 84 & 68.3 & 0.000 \\
\hline Yes & 612 & 83.3 & 300 & 49.0 & 312 & 51.0 & \\
\hline \multicolumn{8}{|c|}{ Eating supper regularly } \\
\hline No & 194 & 26.4 & 65 & 33.5 & 129 & 66.5 & 0.000 \\
\hline Yes & 541 & 73.6 & 274 & 50.6 & 267 & 49.4 & \\
\hline \multicolumn{8}{|l|}{ Snacking between } \\
\hline \multicolumn{8}{|l|}{ breakfast and lunch } \\
\hline Always & 126 & 17.1 & 59 & 46.8 & 67 & 53.2 & 0.686 \\
\hline Sometimes & 477 & 64.9 & 215 & 45.1 & 262 & 54.9 & \\
\hline Never & 132 & 18.0 & 65 & 49.2 & 67 & 50.8 & \\
\hline \multicolumn{8}{|c|}{ Snacking between lunch } \\
\hline \multicolumn{8}{|c|}{ and supper } \\
\hline Always & 236 & 32.1 & 116 & 49.2 & 120 & 50.8 & 0.498 \\
\hline Sometimes & 406 & 55.2 & 180 & 44.3 & 226 & 55.7 & \\
\hline Never & 93 & 12.7 & 43 & 46.2 & 50 & 53.8 & \\
\hline \multicolumn{8}{|l|}{ Midnight snacks } \\
\hline Always & 139 & 18.9 & 62 & 44.6 & 77 & 55.4 & 0.303 \\
\hline Sometimes & 286 & 38.9 & 142 & 49.7 & 144 & 50.3 & \\
\hline Never & 310 & 42.2 & 135 & 43.5 & 175 & 56.5 & \\
\hline
\end{tabular}

*Chi-square test, the $p$-value indicates the comparison between males and females.

The size and frequency of the intake of fast food and sweets by Bahraini adolescents are seen in Table 4. There was no significant difference between gender and frequency of eating fast foods per week. About $14.4 \%$ of adolescents ate fast food daily, while $29 \%$ rarely did so. In general, males were more likely to eat fast foods outside their home than females $(P<0.001)$, whereas females preferred to eat fast foods inside the home $(P<0.01)$. A small percentage $(8.4 \%)$ of adolescents reported not eating burgers and a majority of respondents $(68.8 \%)$ preferred regular size burgers. However, the tendency to eat large size burgers was more pronounced among males than females $(P<0.001)$. A regular portion size of potato chips was preferred by $63.3 \%$ of the adolescents, among them $58.9 \%$ were females and $41.1 \%$ were males $(P<0.002)$. There was a wide range of preferences for soft drinks with $42.2 \%$ of participants consuming them every day and $27.8 \%$ consuming them rarely. Females showed a higher rate in the consumption of small and medium size soft drinks (57.8 and 56.3\%, respectively) compared to males ( 42.2 and $43.7 \%$, respectively) $(P<0.05)$. Females were more prone to consume sweets $(P<0.01)$ and chocolates $(P<0.001)$ than males.

The lifestyle habits of Bahraini adolescents are shown in Table 5. Only a negligible percentage $(5.3 \%)$ did not watch television (TV) daily. There was no significant difference between males and females in watching TV. Of the adolescents, $41.8 \%$ always consumed food while watching TV while only $18.0 \%$ did not. No significant difference was reported between genders in eating while watching TV. More than $50 \%$ of adolescents browsed the Internet for 1 to 2 hours per day and $17.7 \%$ browsed for 3 to 4 hours a day. A very small proportion of adolescents $(6.6 \%)$ ate while browsing the Internet, of whom $68.6 \%$ were males and $31.4 \%$ were females.

Over a quarter $(26.5 \%)$ of the respondents never participated in any sporting activity, with a greater proportion being females $(79.5 \%)$ compared to males $(20.5 \%)$. About $69.2 \%$ of males practiced sports daily as against $30.8 \%$ of females $(P<0.001)$. Participation in sports activities in school was high $(79.9 \%)$ while only $6.1 \%$ never took part in school sports. Amongst the adolescent participants who always engage in sports activities outside school, the majority were males $(81.6 \%)$ while only $18.4 \%$ were females. In general, males significantly participated in more sport activity either at school $(P<0.01)$ or outside school $(P<0.001)$ than females.

\section{Discussion}

This study indicates unfavorable dietary habits and lifestyle by a high proportion of Bahraini adolescents. A previous study in Bahrain reported that about 19 and $22 \%$ of school males and females skipped breakfast, respectively (11). However, it is difficult to compare these findings with the results of this study due to the differences in age group and the way the questions were phrased. A similar tendency for skipping breakfast has been reported among Swedish (13) and Turkish (14) adolescents, where female adolescents were more likely to display abnormal eating attitudes and dieting behaviors than males. For females, skipping breakfast may be connected 
Table 3. Type and frequency of food consumed by Bahraini adolescents

\begin{tabular}{|c|c|c|c|c|c|c|c|}
\hline \multirow{2}{*}{$\begin{array}{l}\text { Frequency of eating } \\
\text { different food/week }\end{array}$} & \multicolumn{2}{|c|}{ Total } & \multicolumn{2}{|c|}{ Male } & \multicolumn{2}{|c|}{ Female } & \multirow[b]{2}{*}{$P$-value } \\
\hline & $N$ & $\%$ & $n$ & $\%$ & $n$ & $\%$ & \\
\hline \multicolumn{8}{|l|}{ Fruits } \\
\hline Daily & 186 & 25.3 & 95 & 51.1 & 91 & 48.9 & 0.000 \\
\hline I-3 times & 238 & 32.4 & 115 & 48.3 & 123 & 51.7 & \\
\hline 46 times & 107 & 14.6 & 60 & 56.1 & 47 & 43.9 & \\
\hline Rarely & 203 & 27.7 & 68 & 33.5 & 135 & 66.5 & 0.001 \\
\hline \multicolumn{8}{|l|}{ Vegetables/salad } \\
\hline Daily & 193 & 26.3 & 102 & 52.8 & 91 & 47.2 & 0.125 \\
\hline I-3 times & 192 & 26.1 & 89 & 46.4 & 103 & 53.6 & \\
\hline 46 times & 70 & 9.5 & 31 & 44.3 & 39 & 55.7 & \\
\hline Rarely & 280 & 38.1 & 117 & 41.8 & 163 & 58.2 & \\
\hline \multicolumn{8}{|c|}{ Milk and dairy products } \\
\hline Daily & 273 & 37.1 & 129 & 47.3 & 144 & 52.7 & 0.017 \\
\hline I-3 times & 198 & 26.9 & 92 & 46.5 & 106 & 53.5 & \\
\hline 46 times & 100 & 13.6 & 57 & 57.0 & 43 & 43.0 & \\
\hline Rarely & 164 & 22.3 & 61 & 37.2 & 103 & 62.8 & \\
\hline \multicolumn{8}{|l|}{ Meat } \\
\hline Daily & 147 & 20.0 & 84 & 57.1 & 63 & 42.9 & 0.000 \\
\hline I-3 times & 305 & 41.5 & 134 & 43.9 & $|7|$ & 56.1 & \\
\hline 46 times & 125 & 17.0 & 67 & 53.6 & 58 & 46.4 & \\
\hline Rarely & 158 & 21.5 & 54 & 34.2 & 104 & 65.8 & \\
\hline \multicolumn{8}{|l|}{ Fish } \\
\hline Daily & 51 & 6.9 & 31 & 60.8 & 20 & 39.2 & 0.003 \\
\hline I-3 times & 284 & 38.6 & 135 & 47.5 & 149 & 52.5 & \\
\hline 46 times & 114 & 15.5 & 62 & 54.4 & 52 & 45.6 & \\
\hline Rarely & 286 & 38.9 & 111 & 38.8 & 175 & 61.2 & \\
\hline \multicolumn{8}{|l|}{ Chicken } \\
\hline Daily & 134 & 18.2 & 74 & 55.2 & 60 & 44.8 & 0.067 \\
\hline I-3 times & 364 & 49.5 & 154 & 42.3 & 210 & 57.7 & \\
\hline 46 times & 172 & 23.4 & 83 & 48.3 & 89 & 51.7 & \\
\hline Rarely & 65 & 8.8 & 28 & 43.1 & 37 & 56.9 & \\
\hline \multicolumn{8}{|l|}{ Lentil/beans } \\
\hline Daily & 30 & 4.1 & 15 & 50.0 & 15 & 50.0 & $0.84 I$ \\
\hline I-3 times & 172 & 23.4 & 80 & 46.5 & 92 & 53.5 & \\
\hline 46 times & 74 & 10.1 & 37 & 50.0 & 37 & 50.0 & \\
\hline Rarely & 459 & 62.4 & 207 & 45.1 & 252 & 54.9 & \\
\hline \multicolumn{8}{|l|}{ Nuts } \\
\hline Daily & 56 & 7.6 & 24 & 42.9 & 32 & 57.1 & 0.144 \\
\hline I-3 times & 198 & 26.9 & 95 & 48.0 & 103 & 52.0 & \\
\hline 46 times & 67 & 9.1 & 39 & 58.2 & 28 & 41.8 & \\
\hline Rarely & 414 & 56.3 & 181 & 43.7 & 233 & 56.3 & \\
\hline \multicolumn{8}{|l|}{ Canned fruit juice } \\
\hline Daily & 176 & 23.9 & 66 & 37.5 & 110 & 62.5 & 0.053 \\
\hline I-3 times & 206 & 28.0 & 104 & 50.5 & 102 & 49.5 & \\
\hline 46 times & 125 & 17.0 & 57 & 45.6 & 68 & 54.4 & \\
\hline Rarely & 228 & 31.0 & 112 & 49.1 & 116 & 50.9 & \\
\hline
\end{tabular}

*Chi-square test, the $p$-value indicates the comparison between males and females.
Table 4. Fast food intake of Bahraini adolescents

\begin{tabular}{|c|c|c|c|c|c|c|c|}
\hline \multirow[b]{2}{*}{ Fast food intake } & \multicolumn{2}{|c|}{ Total } & \multicolumn{2}{|c|}{ Male } & \multicolumn{2}{|c|}{ Female } & \multirow[b]{2}{*}{$P$-value* } \\
\hline & $N$ & $\%$ & $n$ & $\%$ & $n$ & $\%$ & \\
\hline \multicolumn{8}{|c|}{ Frequency of eating fast } \\
\hline \multicolumn{8}{|l|}{ food/week } \\
\hline Daily & 106 & 14.4 & 52 & 49.1 & 54 & 50.9 & .327 \\
\hline $\mathrm{I}-3$ times & 277 & 37.7 & 132 & 47.7 & 145 & 52.3 & \\
\hline 46 times & 139 & 18.9 & 68 & 48.9 & 71 & 51.1 & \\
\hline Rarely or not & 213 & 29.0 & 87 & 40.8 & 126 & 59.2 & \\
\hline \multicolumn{8}{|c|}{ Frequency of eating fast } \\
\hline \multicolumn{8}{|c|}{ food outside home/week } \\
\hline Never & 133 & 18.1 & 49 & 36.8 & 84 & 63.2 & 0.000 \\
\hline Once & 153 & 20.8 & 55 & 35.9 & 98 & 64.1 & \\
\hline Twice & $17 \mid$ & 23.3 & 66 & 38.6 & 105 & 61.4 & \\
\hline Three times & $|3|$ & 17.8 & 76 & 58.0 & 55 & 42.0 & \\
\hline Four times + & 147 & 20.0 & 93 & 63.3 & 54 & 36.7 & \\
\hline \multicolumn{8}{|c|}{ Frequency of eating fast } \\
\hline \multicolumn{8}{|c|}{ food inside home/week } \\
\hline Never & 126 & 17.2 & 77 & 61.1 & 49 & 38.9 & 0.006 \\
\hline One time & 136 & 18.5 & 60 & 44.1 & 76 & 55.9 & \\
\hline Two times & 166 & 22.6 & 75 & 45.2 & 91 & 54.8 & \\
\hline Three times & 101 & 13.8 & 44 & 43.6 & 57 & 56.4 & \\
\hline Four times + & 205 & 27.9 & 83 & 40.5 & 122 & 59.5 & \\
\hline \multicolumn{8}{|c|}{ Size of burger preferred } \\
\hline Don't eat & 62 & 8.4 & 25 & 40.3 & 37 & 59.7 & 0.000 \\
\hline Regular & 506 & 68.8 & 208 & 41.1 & 298 & 58.9 & \\
\hline Large & 167 & 22.7 & 106 & 63.5 & 61 & 36.5 & \\
\hline \multicolumn{8}{|c|}{ Size of chips preferred } \\
\hline Don't eat & 90 & 12.2 & 52 & 57.8 & 38 & 42.2 & 0.002 \\
\hline Regular & 465 & 63.3 & 191 & $4 I . I$ & 274 & 58.9 & \\
\hline Large & 179 & 24.4 & 95 & 53.1 & 84 & 46.9 & \\
\hline \multicolumn{8}{|l|}{ Soft drinks } \\
\hline Daily & 310 & 42.2 & 175 & 56.5 & 135 & 43.5 & 0.000 \\
\hline $\mathrm{I}-3$ times & 130 & 17.7 & 50 & 38.5 & 80 & 61.5 & \\
\hline 46 times & 91 & 12.4 & 45 & 49.5 & 46 & 50.5 & \\
\hline Rarely or not & 204 & 27.8 & 69 & 33.8 & 135 & 66.2 & \\
\hline \multicolumn{8}{|l|}{ Size of soft drinks } \\
\hline \multicolumn{8}{|l|}{ preferred } \\
\hline Don't drink & 85 & 11.6 & 45 & 52.9 & 40 & 47.1 & 0.036 \\
\hline Small & 270 & 36.7 & 114 & 42.2 & 156 & 57.8 & \\
\hline Medium & 268 & 36.5 & 117 & 43.7 & $15 \mid$ & 56.3 & \\
\hline Large & 112 & 15.2 & 63 & 56.3 & 49 & 43.8 & \\
\hline \multicolumn{8}{|c|}{ Intake of sweets/week } \\
\hline Daily & 231 & 31.4 & 86 & 37.2 & 145 & 62.8 & 0.003 \\
\hline $\mathrm{I}-3$ times & 193 & 26.3 & 93 & 48.2 & 100 & 51.8 & \\
\hline 46 times & 105 & 14.3 & 47 & 44.8 & 58 & 55.2 & \\
\hline Rarely & 206 & 28.0 & 113 & 54.9 & 93 & 45.1 & \\
\hline \multirow{2}{*}{\multicolumn{8}{|c|}{$\begin{array}{l}\text { Intake of chocolates/ } \\
\text { week }\end{array}$}} \\
\hline & & & & & & & \\
\hline Daily & 239 & 32.5 & 85 & 35.6 & 154 & 64.4 & 0.000 \\
\hline $\mathrm{I}-3$ times & 191 & 26.0 & 92 & 48.2 & 99 & 51.8 & \\
\hline 46 times & 95 & 12.9 & 45 & 47.4 & 50 & 52.6 & \\
\hline Rarely & 210 & 28.6 & 117 & 55.7 & 93 & 44.3 & \\
\hline
\end{tabular}

*Chi-square test, the $p$-value indicates the comparison between males and females. 
Table 5. Lifestyle habits amongst Bahraini adolescents

\begin{tabular}{|c|c|c|c|c|c|c|c|}
\hline \multirow[b]{3}{*}{ Lifestyle habits } & \multicolumn{7}{|c|}{ Gender } \\
\hline & \multicolumn{2}{|c|}{ Total } & \multicolumn{2}{|c|}{ Male } & \multicolumn{2}{|c|}{ Female } & \multirow[b]{2}{*}{$P$-value } \\
\hline & $N$ & $\%$ & $n$ & $\%$ & $n$ & $\%$ & \\
\hline \multicolumn{8}{|l|}{$\begin{array}{l}\text { Hours watching TV } \\
\text { every day }\end{array}$} \\
\hline \multicolumn{8}{|l|}{ rarely } \\
\hline I-2 hours & 219 & 29.8 & 101 & 46.1 & 118 & 53.9 & \\
\hline 3-4 hours & 270 & 36.7 & 121 & 44.8 & 149 & 55.2 & \\
\hline $5+$ hours & 207 & 28.2 & 101 & 48.8 & 106 & 51.2 & \\
\hline \multicolumn{8}{|l|}{ Eating while watching } \\
\hline \multicolumn{8}{|l|}{ TV } \\
\hline Always & 307 & 41.8 & 135 & 44.0 & 172 & 56.0 & 0.145 \\
\hline Sometimes & 296 & 40.3 & 133 & 44.9 & 163 & 55.1 & \\
\hline Never & 132 & 18.0 & 7I & 53.8 & 61 & 46.2 & \\
\hline \multicolumn{8}{|l|}{$\begin{array}{l}\text { Hours using Internet } \\
\text { every day }\end{array}$} \\
\hline Not using & 202 & 27.5 & 87 & 43.1 & 115 & 56.9 & 0.095 \\
\hline I-2 hours & 403 & 54.8 & 200 & 49.6 & 203 & 50.4 & \\
\hline 3-4 hours & 130 & 17.7 & 52 & 40.0 & 78 & 60.0 & \\
\hline \multicolumn{8}{|l|}{ Eating while using } \\
\hline \multicolumn{8}{|l|}{ Internet } \\
\hline Don't eat & 498 & 93.4 & 241 & 48.4 & 257 & 51.6 & 0.052 \\
\hline Eat & 35 & 6.6 & II & 31.4 & 24 & 68.8 & \\
\hline \multicolumn{8}{|c|}{$\begin{array}{l}\text { Participating in sporting } \\
\text { activities }\end{array}$} \\
\hline No & 195 & 26.5 & 41 & 21.0 & 154 & 79.0 & 0.000 \\
\hline Yes & 540 & 73.5 & 298 & 55.2 & 242 & 44.8 & \\
\hline \multicolumn{8}{|l|}{$\begin{array}{l}\text { Frequency of partici- } \\
\text { pating sport/week }\end{array}$} \\
\hline None & 195 & 26.5 & 40 & 20.5 & 155 & 79.5 & 0.000 \\
\hline $\mathrm{I}-2$ & 167 & 22.7 & 62 & 37.1 & 105 & 62.9 & \\
\hline $3-4$ & 168 & 22.9 & 94 & 56.0 & 74 & 44.0 & \\
\hline $5-6$ & 72 & 9.8 & 51 & 70.8 & 21 & 29.2 & \\
\hline $7+$ & 133 & 18.1 & 92 & 69.2 & 41 & 30.8 & \\
\hline \multicolumn{8}{|l|}{$\begin{array}{l}\text { Participating in sports } \\
\text { activity at school }\end{array}$} \\
\hline Always & 587 & 79.9 & 268 & 45.7 & 319 & 54.3 & 0.026 \\
\hline Sometimes & 103 & 14.0 & 42 & 40.8 & 61 & 59.2 & \\
\hline Never & 45 & 6.1 & 29 & 64.4 & 16 & 35.6 & \\
\hline \multicolumn{8}{|c|}{$\begin{array}{l}\text { Participating in sports } \\
\text { activities outside school }\end{array}$} \\
\hline Always & 266 & 36.2 & 217 & 81.6 & 49 & 18.4 & 0.000 \\
\hline Sometimes & 298 & 40.5 & 88 & 29.5 & 210 & 70.5 & \\
\hline Never & 171 & 23.3 & 34 & 19.9 & 137 & 80.1 & \\
\hline
\end{tabular}

*Chi-square test, the $p$-value indicates the comparison between males and females. in some way to feelings about body shape and it may also be a method of dieting. For males, however, skipping breakfast may be associated with lack of time or accessibility (15). Skipping breakfast may be related to risk for obesity and cardio-metabolic health. In a longitudinal study, Smith et al. (16) found that participants who skipped breakfast in both childhood and adulthood had a larger waist circumference and higher fasting insulin, total cholesterol, and LDL cholesterol concentrations than those who ate breakfast regularly. In addition, regular breakfast intake has a positive association with attention-concentration, memory, and school achievement among school children (17). Although breakfast was often skipped, we found that lunch and dinner were consumed regularly by most of the adolescents. A similar pattern of food intake has been reported amongst adolescents in Nordic countries (13) where the skipping of breakfast was reported, but about $80 \%$ of the adolescents consumed a regular afternoon meal and dinner.

It has been documented that boys and girls with irregular breakfast intake had received a high percentage of their energy needs from in-between meals (18). Musiager et al. (19) reported that some foods commonly provided by school canteens in Bahrain have a greater caloric value. In addition, in a study describing the contribution of school meals to nutrient intake amongst English primary and secondary schools, it was reported that school meals often failed to make good the shortfalls in the daily intake of essential nutrients (20). The frequent consumption of snacks is a recognized aspect of teenage food behavior. The finding that a greater proportion of adolescents consumed snacks is similar to findings amongst Syrian adolescents, where snacking and light meal consumption was very common (21).

The present study showed that about $25 \%$ of total adolescent consumed fruit and vegetables daily. Low intake of fruit and vegetables is associated with several chronic diseases at adulthood (22). In the United States Krebs-Smith et al. (23) found that among children aged 2-18 years, mean consumption of fruit and vegetables was 3.5 servings per day with consumption increasing in older males ( 4.3 servings per day), but remaining the same among females regardless of age. In general, although the proportion of males who consumed fruit was higher than females, vegetable consumption was higher amongst females. Studies on fruit and vegetable consumption among children indicate that some of the determinants that influence consumption are gender, parental intake, and home availability/accessibility (24).

Fast food has become a prominent feature of the diet of children throughout the world (25). Our study showed a relatively high intake of fast foods by adolescents 
in Bahrain. This is especially true when we compare our findings with those previously reported by Musaiger and Gregory (11) where none of the school children mentioned that they consumed fast foods. Studies in Arab Gulf countries (5-7) have shown that fast foods are now becoming an integral part of the lifestyle of school children. The fact that girls consume fast foods more at home than the boys could be due to cultural restrictions on travel and ease of home delivery from fast food restaurants that have become very popular in Bahrain in the past few years, especially among the younger generation. The preference of males for larger portions of burgers and potato chips is similar to that reported amongst Kuwaiti adolescents, where males were more likely than females to consume larger size portions of fast foods (26).

While watching television TV has profoundly changed the use of leisure time in many countries, there is evidence that it is associated with negative outcomes such as poor dietary habits (27). In addition, in recent years, computers have begun to play a vital role in today's generation as the need for information has increased (28). There is mounting evidence that excessive TVviewing among children and adolescents can seriously challenge young people's emotional and physical well-being (29). Our study indicates that a good number of adolescents spend a substantial portion of their time watching TV or surfing the Internet every day. While there are potential benefits from watching some TV programs and using the Internet, studies amongst preschoolers indicate that greater exposure to TV and videos may influence the consumption of unhealthy foods (30). Although the proportion of adolescents who eat while using the Internet in this study was low, snacking while watching TV - especially amongst girls - was high. Snacking, but not necessarily eating meals, while watching $\mathrm{TV}$ is associated with increased overall caloric intake and calories from fat (31). It was found that overweight and obesity among school children are directly related to the amount of time spent in front of a TV set or personal computer $(p<0.01)(32)$.

In children and adolescents alike, maintaining an optimal level of physical activity is particularly important as it not only reduces the risk of overweight but also improves body composition and the growth pattern (33). In this study we found that the greater proportion of adolescents who did not participate in sports was females, while a considerable percentage of males participated in physical activity. The exact nature and duration of activity could not be ascertained owing to the fact that students encountered difficulties in describing the activity correctly. Gender difference was significant with regard to practicing sports during school hours and also outside school; however, females were less active than males. Similar findings are reported from other Arabian Gulf countries $(34,35)$. One of the main reasons for girls not participating in sports can be social and religious norms and restrictions that may preclude female students from engaging in public sports (36). This cultural drawback further contributes toward the rising epidemic of overweight and obesity prevalent in the Arabian Gulf countries.

In conclusion, adolescents in Bahrain seem to be moving toward unhealthy eating habits in conjunction with a diet high in saturated fats, sugar, and refined foods but low in fiber often termed the 'Western diet' and on lifestyles characterized by lower levels of activity (37). This may increase the risk factors for chronic noncommunicable diseases in a later age such as coronary heart disease, diabetes, hypertension, and cancer. These diseases have become the main public health problems in most of the countries in the Middle East (38). Nutrition education among both school children and their parents should emphasize the importance of following dietary guidelines recently developed for the Arab countries (39). Regular interaction between parents, adolescents, school authorities, and health personnel is required to emphasize the connection between health, healthy food choices, and lifestyle habits.

\section{Conflict of interest and funding}

The authors have not received any funding or benefits from industry or elsewhere to conduct this study.

\section{References}

1. Lytle LA, Seifert S, Greenstein J, McGovern P. How do children's eating patterns and food choice change over time? Results from a cohort study. Am J Health Promotion 2000; 14: 222-8.

2. Story M, Neumark-Sztainer D, French S. Individual and environmental influences on adolescent eating behaviors. J Am Dietetic Assoc 2002; 102: S40-51.

3. Hilsen M, Eikemo TA, Bere E. Healthy and unhealthy eating at lower secondary school in Norway. Scand J Public Health 2010; 38: 7-12.

4. Rolland-Cachera MF, Bellsile F, Deheeger M. Nutritional status and food intake in adolescents living in Western Europe. Eur J Clin Nutr 2000; 54: S41-6.

5. Qotba H, Al-Isa AN. Anthropometric measurements and dietary habits of school children in Qatar. Int J Food Sci Nutr 2007; 58: $1-5$.

6. Bin Zaal AA, Musaiger AO, D'Souza R. Dietary habits associated with obesity among adolescents in Dubai, United Arab Emirates. Nutr Hosp 2009; 24: 437-44.

7. Washi SA, Ageib MB. Poor diet quality and food habits are related to impaired nutritional status in 13- to 18-year old adolescents in Jeddah. Nutr Res 2010; 30: 527-34.

8. El-Hazmi MAF, Warsy AS. Association of hypertension and non-insulin-dependent diabetes mellitus in the Saudi population. Ann Saudi Med 2001; 21: 5-8.

9. Musaiger AO. Nutritional status and dietary habits of adolescent girls in Oman. Ecology Food Nutr 1994; 31: 227-37.

10. Musaiger AO. Overweight and obesity in the Eastern Mediterranean Region: can we control it? East Mediterr Health J 2004; 10: 789-93. 
11. Musaiger AO, Gregory WB. Dietary habits of school-children in Bahrain. J Royal Soc Health 1992; 112: 159-62.

12. Al-Sendi AM. Factors determining obesity among adolescents in Bahrain. PhD Dissertation, London School of Tropical Medicine; 2002.

13. Samuelson G. Dietary habits and nutritional status in adolescents over Europe. An overview of current studies in the Nordic countries. Eur J Clin Nutr 2000; 54: S21-8.

14. Baş M, Kiziltan G. Relations among weight control behaviors and eating attitudes, social physique anxiety, and fruit and vegetable consumption in Turkish adolescents. Adolescence 2007; 42: 167-78.

15. Shaw ME. Adolescent breakfast skipping: an Australian study. Adolescence 1998; 33: 851-61.

16. Smith KJ, Gall SL, McNaughton SA, Bizzard L, Dwyer T, Venn AJ. Skipping breakfast: longitudinal associations with cardiometabolic risk factors in the Childhood Determinants of Adult Health Study. Am J Clin Nutr 2010; 92: 1316-25.

17. Gajre NS, Fernandez S, Balekrishna N, Vazir S. Breakfast eating habit and its influence on attention-concentration, immediate memory and school achievement. Indian Pediatr 2008; 45: 824-8.

18. Sjoberg A, Hallberg L, Hoglund D, Hulthen L. Meal pattern, food choice, nutrient intake and lifestyle factors in the Goteborg Adolescence Study. Eur J Clin Nutr 2003; 57: 1569-78.

19. Musaiger AO, Al-Jedah JH, D’Souza R. Nutritional profile of ready-to-eat foods consumed in Bahrain. Ecology Food Nutr 2007; 46: 47-60.

20. Nelson M, Lowes K, Hwang V. The contribution of school meals to food consumption and nutrient intakes of young people aged 4-18 years in England. Public Health Nutr 2007; 10: $652-62$.

21. Lock K, Pomerleau J, Causer L, Altmann DR, Mckee M. The global burden of disease attributable to low consumption of fruit and vegetables: implications for the global strategy on diet. Bull World Health Organ 2005; 83: 100-8.

22. Kerkadi A. Evaluation of nutritional status of United Arab Emirates university female students. Emirates J Agricultural Sci 2003; 15: 42-50; http://www.cfs.uaeu.ac.ae/research/ejas.html. [cited 20 January 2011]

23. Krebs-Smith SM, Cook DA, Subar AF, Cleveland L, Friday J, Kahle LL. Fruit and vegetable intakes of children and adolescents in the United States. Arch Pediatr Adolesc Med 1996; 150: $81-6$.

24. Rasmussen F, Johansson M. The relation of weight, length and ponderal index at birth to body mass index and overweight among 18-year-old males in Sweden. Eur J Epidemiol 1998; 14: 373-80.

25. Bowman SA, Gortmaker SL, Ebbeling CB, Pereira MA, Ludwig DS. Effects of fast-food consumption on energy intake and diet quality among children in a national household survey. Pediatrics 2004; 113: 112-8.

26. Al-Mulla D, Al-Najdi F, Al-Aazmi M, Al-Serri M, Badoo M, Othman R, et al. Knowledge and practices related to obesity among university students in Kuwait. Paper presented at the 10th International Congress on Obesity, Sydney, Australia, 3-8 September 2006.

27. Jeffery RW, French SA. Epidemic obesity in the United States: are fast foods and television viewing contributing? Am J Public Health 1998; 88: 277-80.

28. Stefănescu C, Chele G, Chiriță V, Chiriță R, Mavros M, Macarie G, et al. The effects of computer-use on adolescents [in Romanian]. Revista Medico-Chiruricala A Societatii de Medici si Naturalisti Din Iasi (Iasi) 2005; 109: 871-7.

29. Hardy LL, Baur LA, Garnett SP, Crawford D, Campbell KJ, Shrewsbury VA, et al. Family and home correlates of television viewing in 12-13 year old adolescents: The Nepean Study. Int J Behav Nutr Phys Activity 2006; 3: 24.

30. Taveras EM, Sandora TJ, Shih M-C, Ross-Degnan D, Goldmann DA, Gillman MW. The association of television and video viewing with fast food intake by preschool-age children. Obesity 2006; 14: 2034-41.

31. Gore SA, Foster JA, DiLillo VG, Kirk K, Smith West D. Television viewing and snacking. Eating Behav 2003; 4: 399-405.

32. Ramic E, Kapidzic-Durakovic S, Karic E, Batic-Mujanoric O, Alibasic E, Zildzic M. Influence of lifestyle on overweight and obesity in school-age children. Med Arh 2009; 63: 280-3.

33. Deheeger M, Rolland-Cachera MF, Fontvielle AM. Physical activity and body composition in 10 year old French children: linkages with nutritional intake? International J Obes Related Metab Disord 1997; 21: 372-9.

34. Amine EK, Samy M. Obesity among female university students in the United Arab Emirates. J Royal Soc Health 2000; 116: $91-6$.

35. Rasheed P. Overweight status: body image \& weight control beliefs and practices among female college students. Ann Saudi Med 1999; 19: 365-9.

36. Henry CJK, Lightowler HJ, Al-Hourani HM. Physical activity and level of inactivity in adolescent females aged 11-16 years in the United Arab Emirates. Am J Hum Biol 2004; 16: 346-53.

37. Popkin BM, Gordon-Larsen P. The nutrition transition: worldwide obesity dynamics and their determinants. Int J Obes 2004; 28: S2-9.

38. Musaiger AO. Diet and prevention of coronary heart disease in the Arab Middle East Countries. Med Princ Pract 2002; 11: 916.

39. Arab Center for Nutrition. The food dome: dietary guidelines for the Arab Countries; http://www.acnut.com/en/view.php?id=2; 2005.

\footnotetext{
*Abdulrahman O. Musaiger

Director of Nutrition and Health Studies Unit

Deanship of Scientific Research

University of Bahrain

Kingdom of Bahrain

Email: amusaiger@gmail.com
} 